\title{
Erratum
}

\section{Rational points of bounded height on Fano varieties}

\section{Jens Franke $^{1}$, Yuri I. Manin ${ }^{2}$, and Yuri Tschinkel ${ }^{3}$}

${ }^{1}$ Universität Jena, Sekt. Math., Universitäts-Hochhaus 17.OG, DDR-6900 Jena and Dept. of Math., Moscow University

${ }^{2}$ Steklov Math. Institute, 42 Vavilova, 117966 GSP-1, Moscow, USSR

${ }^{3}$ Dept. of Math., Moscow University, 117234, Moscow, USSR

Invent. Math. 95, 421-435 (1989)

The formula on p. 432 describing the leading term of the asymptotic for rational points on the flag manifold of a split group, with the height function normalised by a Chevalley lattice, was misprinted. The correct formula is

$$
\lim _{s \rightarrow 1}(s-1)^{\mathrm{rk}(P)} Z_{-2 \rho}(s)=\prod_{\alpha \in \Phi_{P}-\Delta_{0}} \frac{\xi\left(\left\langle\check{\alpha}, \rho_{P_{0}}\right\rangle\right)}{\xi\left(\left\langle\check{\alpha}, \rho_{P_{0}}\right\rangle+1\right)} \prod_{\alpha \in \Delta_{0}-\Delta_{0}^{P}} \frac{\operatorname{Res}_{s=1} \xi}{\xi(2)\left\langle\check{\alpha}, 2 \rho_{P}\right\rangle} .
$$

The misprinted formula had the subscripts ' 0 ' at $\rho_{P_{0}}$ omitted. The resulting expression is syntactically correct, but gives a wrong result. 